\title{
El enfoque morfogenético de Margaret Archer para el análisis de la cultura
}

\author{
The morphogenetic approach of Margaret Archer for the analysis of culture
}

Yasmín Hernández-Romero (yasmin_h_r@hotmail.com) Centro Universitario Zumpango, Universidad Autónoma del Estado de México (Estado de México, México) ORCID: 0000-0002-2235-3611

\begin{abstract}
Margaret Archer has elaborated a proposal for the analysis of culture, known as morphogenetic approach, whose axis runs parallel to the classical debate within the social theory on the structure-agency relation. Archer's perspective is shaped by what she calls analytic dualism, which is presented as an alternative to the reductionist stances that emphasize a single dimension (called structure or agency), but her perspective also differs from the underlying integrationist stance in the theory of structuring. To elucidate the morphogenetic approach, the postulates of critical realism are explained, with the ontological and epistemological foundations of which it is linked. The contribution made by Archer proves to be a necessary reference for the approach and explanation of the cultural dynamics because it allows to identify elements to explain why some contradictions produce changes and others do not.
\end{abstract}

Key words: Margaret Archer, culture, morphogenetic approach, critical realism, analytical dualism.

\section{Resumen}

Margaret Archer elabora una propuesta para el análisis de la cultura, conocida como enfoque morfogenético, cuyo eje corre paralelo al debate clásico dentro de la teoría social sobre la relación estructura-agencia. La perspectiva de Archer se configura a partir de lo que llama dualismo analítico, el cual se presenta como una alternativa a las posturas reduccionistas que enfatizan una sola dimensión (llámese estructura o agencia), pero también se diferencia de la postura integracionista subyacente en la teoría de la estructuración. Para elucidar el enfoque morfogenético se explicitan los postulados del realismo crítico, con cuyos fundamentos ontológicos y epistemológicos se vincula. La contribución realizada por Archer resulta ser un referente obligado para el abordaje y explicación de la dinámica cultural en tanto que, permite identificar elementos a partir de los cuales explicar por qué algunas contradicciones producen cambios y otras no lo hacen.

Palabras clave: Margaret Archer, cultura, enfoque morfogenético, realismo crítico, dualismo analítico.

\section{Introducción}

Margaret Archer es una de las grandes exponentes de la teoría sociológica contemporánea, al lado de teóricos como Bourdieu, Giddens y Habermas. Su planteamiento teórico se sitúa dentro del debate iniciado durante la década de 1980 en torno a la relación estructura-agencia, debate del cual, en términos generales, se han derivado propuestas integracionistas que intentan superar el reduccionismo de los enfoques estructuralista y voluntarista de la acción. En este contexto, la singularidad del planteamiento 
de la socióloga británica se configura a partir de la discusión que establece con los enfoques reduccionistas antes mencionados, y con la teoría de la estructuración, de Anthony Giddens. Para Archer, la dualidad entre agencia y estructura, que propone Giddens para resolver la dicotomía mencionada, termina elidiendo las diferencias entre ambas. Archer llama a su propuesta el dualismo analítico, en el que el problema de la agencia y estructura si bien mantienen relaciones de interdependencia, se deshacen con fines analíticos y se observan en diferentes momentos, como teóricamente distintos; pero también se analizan en su interacción.

Pese a la relevancia teórica de los planteamientos de Archer, sus aportes han sido insuficientemente divulgados en el ámbito académico de habla hispana. Por lo que el presente documento pretende contribuir a la difusión del pensamiento de una de las intelectuales más representativas de la sociología contemporánea.

Margaret Archer nació en Grenoside, Reino Unido, el 20 de enero de 1943. Estudió sociología en la London School of Economics and Political Science y la École Normale Supérieure, en París, de la cual se graduó en 1964. Se doctoró en la misma escuela en 1967, con la tesis: The educational achievement aspirations of English working-class parents: their formation and influence on children's school. Sus estudios de postdoctorado los realizó en la Sorbona de París.

Trabajó como profesora de la Universidad de Reading, y fue profesora de sociología en la Universidad de Warwick desde 1979 hasta el año de 2010. Actualmente es catedrática de la Escuela Politécnica Federal de Lausanne, en Suiza. En esta última también ocupó el puesto de directora del Centro de Ontología Social. Entre otras de sus actividades destacables se encuentran: su trabajo como editora de la Revista Current Sociology, de la Asociación Internacional de Sociología, desarrollado de 1971 a 1980; haber sido fundadora de la Academia de Ciencias Sociales en el Reino Unido e integrante de la Academia Europea, y Codirectora del Centro de Realismo Crítico. Durante el periodo de 1986 a 1990 fue presidenta de la Asociación Internacional de Sociología, siendo la primera mujer en ocupar dicho cargo. En 1994 participó como miembro fundador de la Academia Pontifica de Ciencias Sociales, y es nombrada presidenta de dicha Academia en abril de 2014.

Dentro de sus obras se encuentran: Social origins of educational systems (1979), Culture and agency: the place of culture in social theory (1988), Realist social theory: the morphogenetic approach (1995), Being human: the problem of agency (2000), Structure, agency and the internal conversation (2003), Making our way through the world: human reflexivity and social mobility (2007), Conversations about reflexivity (2010), The reflexive imperative in late modernity (2012). Además de diversos artículos en revistas especializadas y capítulos de libros, como por ejemplo los tres capítulos que presenta en el libro Critical realism. Essential readings (1998), libro del que fue coordinadora junto a Roy Bhaskar, Andrew Collier, Tony Lawson y Alan Norrie.

Como se puede apreciar, el trabajo teórico de Archer ha estado centrado, en su producción intelectual de las últimas tres décadas, en el debate teórico acerca de la estructura y la agencia. Archer ubica este debate junto al de la relación cultura y agencia, el cual constituye el eje de su explicación de la cultura. Sin embargo, bajo este enfoque, la cultura no se subsume en la estructura, al ser dos ámbitos diferentes en términos teóricos.

Para Archer, el análisis de la cultura, dentro de la sociología, se ha abordado desde enfoques reduccionistas, y señala que, o bien se ha considerado como una variable independiente, que al "orquestar 
toda la estructura social" (1988:28); o ha quedado reducida a un epifenómeno o reflejo de las estructuras. Inclusive, las propuestas que postulan una perspectiva integracionista no profundizan en cómo abordar el análisis de la cultura, ni en poder captar aquellos elementos que den cuenta de la dinámica cultural.

Algunas de las interrogantes que condujeron la investigación para lo expuesto en este documento son: ¿en qué perspectiva epistemológica, teórica y metodológica se ubica la propuesta de Archer?, ¿con qué conceptualizaciones de la cultura discute?, ¿en qué consiste dicha propuesta teórica-metodológica?, y ¿cuáles son las ventajas que tiene el enfoque por ella sugerido? De esta manera, este texto se encuentra organizado en cuatro apartados. En el primero se presenta una breve descripción del realismo crítico, con la intención de ubicar los presupuestos ontológicos y epistemológicos en que se asienta la teoría de Archer. En el segundo, se plantea cómo el dualismo analítico y el enfoque morfogenético se constituyen en una alternativa a las formas en que, dentro de la teoría social, ha sido abordada la relación estructura-agencia. En el tercero, se muestran los enfoques de la cultura presentes en la sociología, los cuales son el referente de discusión de Archer. En el cuarto, se expone el enfoque morfogenético, con el que Archer explica la dinámica cultural. Finalmente, se presentan algunas reflexiones sobre el tema expuesto.

\section{El realismo crítico como fundamento de la teoría y metodología de Archer}

Si bien las teorías son realizadas por mujeres y hombres con una situación biográfica particular, la producción del conocimiento científico es de carácter social. Es decir, que detrás de toda propuesta existen referentes filosóficos y teóricos que las influyen o articulan, en conjunción con el ambiente social e intelectual de la época.

En este apartado se establece la concepción de realidad social del realismo crítico, al ser la postura ontológica con lo que se vincula la propuesta de Archer, movimiento filosófico del que la propia Archer es parte. Una descripción amplia de esta postura rebasa el propósito de este documento.

El realismo crítico es una postura filosófica, fundada por el filósofo inglés de origen hindú Roy Bhaskar, sobre la realidad (ontología) y el conocimiento humano (epistemología), que pretende ser una alternativa al positivismo y la hermenéutica. En el nivel ontológico, considera que la realidad existe, nos precede, independientemente del conocimiento que se tenga de ella. A este carácter de independencia ontológica de algunas entidades de la realidad se le denomina intransitividad. Empero, la perspectiva de Bhaskar es de un realismo crítico distinto al naturalismo positivista, puesto que la realidad se concibe conformada por estructuras múltiples, ordenadas en cada situación, algunas de las cuales se manifiestan en la superficie y otras se encuentran en un nivel más profundo. En el ámbito de las ciencias sociales, el realismo crítico asume una concepción dinámica del sujeto, derivada del propio objeto de estudio de estas ciencias. Mientras que, para el naturalismo positivista la realidad se encuentra determinada por leyes universales que actúan al margen de la voluntad del sujeto.

"Contrario al positivismo, el realismo crítico afirma que 1) las estructuras sociales son actividadesdependientes (implican seres humanos actuando, haciendo cosas); 2) las estructuras sociales son conceptos-dependientes, lo que significa que se basan también en ideas. Es más, estas estructuras sociales son específicas y geo-históricamente dependientes (variante tiempo-espacio)" (Parada 2004:419).

A diferencia del paradigma hermenéutico, que entiende a la realidad como significativa e intencional y en la que los individuos son los únicos que pueden darle intencionalidad, el realismo crítico reconoce que las estructuras existen, pero que los individuos no se encuentran predeterminados por ellas. Estas estructuras 
se reproducen o transforman a partir de las relaciones sociales. Así, para el realismo crítico, la realidad mantiene un carácter abierto porque está poblada, y los individuos le dan intencionalidad a partir de los procesos de interacción con otros individuos y con las estructuras sociales.

Epistemológicamente, la idea de realidad estructurada por niveles conlleva la necesidad de trascender el nivel empírico de la realidad y acceder al conocimiento de ésta en un nivel de mayor profundidad, buscando los mecanismos causales a partir de los cuales se configura. De esta manera: "el vivir en un mundo de sistemas abiertos tiene, por ende, repercusiones epistemológicas que afectan directamente la posibilidad de aplicar lógicas inductivas o deductivas al análisis social. Éstas se manifiestan en una noción de causalidad que contempla la posibilidad de la incidencia de lo contingente, lo imprevisto" (Parra 2016:221).

Para el realismo crítico el conocimiento de la realidad es aproximado y cambiante, en tanto que es un producto social y, por ende, tiene un carácter transitivo. Lo que supone un relativismo epistemológico. En ese sentido, las afirmaciones cognitivas que se hagan están relacionadas con lo que sabemos en ese momento y con el lugar en que estamos posicionados, lo que les da una forma provisional. Sin embargo, la ciencia tiene como propósito buscar el conocimiento de las estructuras y mecanismos causales reales. Este punto es importante debido a que, si bien la conciencia que tengan los sujetos sobre la realidad es independiente a la existencia de ésta -contrario a la postura hermenéutica-, la conciencia de los procesos sociales que la producen constituye una condición para su transformación. Y, para Bhaskar, el esclarecimiento y descripción de los procesos sociales que configuran la realidad es una tarea de las ciencias sociales.

Se puede resaltar, como colofón a este apartado, que el realismo crítico sostiene una clara postura política de carácter emancipador, presentándose como una alternativa al nuevo realismo o realismo empirista que se acomoda a las formas de la sociedad capitalista actual, que vacía el mundo social de cualquier dimensión estructural perdurable, y no reconoce que hay mecanismos generativos de cambio y transformación.

\section{El dualismo analítico y el enfoque morfogenético de la relación estructura-agencia}

En este apartado se presenta la revisión que Archer hace acerca de las teorías existentes para analizar la relación estructura-agencia, que la lleva a proponer un nuevo enfoque. Para Archer, los modelos explicativos de la acción que se han desarrollado dentro de la teoría social tienen una limitante, y es que son conflacionistas, lo que significa que uno de los momentos se funde en el otro o, en su defecto, se da la elisión entre ambos. La siguiente cita expresa la caracterización que hace Archer de los tres modelos de la acción: "Margaret Archer ha denominado correlativamente a estos modelos como conflacionismo ascendente, conflacionismo descendente y conflacionismo central. Por conflacionismo entiende la anulación del carácter autónomo de la acción, de la estructura o de ambas, es decir, la integración -y con ellos la pérdida o fusión- de la autonomía de la acción en la estructura, de la autonomía de la estructura en la acción, o de ambas en un tercer elemento, como sucede con las prácticas sociales en Giddens. Para hacer frente a esta problemática, Archer ha propuesto un cuarto tipo de relación, la dualistamorfogenética, que mantiene la autonomía de acción y estructura tanto a nivel de explanans como del explanandum y explica lo social como emergencia" (Mascareño 2008:224-225).

Las formas conflacionistas ascendente o descendente pierden el carácter estratificado de la realidad, al considerar una sola dimensión de ésta. La teoría de la acción racional expresa el conflacionismo ascendente, en el sentido de explicar la acción social a partir de la intencionalidad y racionalidad del actor, 
y que, por cierto, lo reduce a un hombre económico. Del otro lado estarían las teorías estructuralistas, o teorías conflacionistas descendentes, que dan primacía al condicionamiento estructural en la explicación de la acción, asumiendo una idea de sujeto sujetado a las estructuras.

En lo que respecta al conflacionismo central o elisionismo, representado por la teoría de la estructuración de Giddens, éste se opone a las visiones dualistas que representan, por un lado, el determinismo estructural, y por el otro, el voluntarismo, proponiendo en su lugar la dualidad de la estructura. Esto es, las estructuras se integran por medio de la acción, y la acción se constituye estructuralmente. En este modelo explicativo ambas dimensiones se presentan como unidad, al ubicarlas simultáneamente en el tiempo, elidiendo la especificidad y temporalidad de cada una de ellas.

La siguiente cita esclarece la noción de dualidad formulada por Giddens: "No hay, entonces, agentes cuya configuración sea independiente de la estructura; esto es, no hay más agentes que los constituidos en el proceso de estructuración de la dualidad de la estructura. No existe una estructura dada, preexistente, ajena a la actividad de los actores, como tampoco existe una acción subjetiva, inteligible unilateralmente. A partir de que se postula esa dualidad, se sostiene la idea de que estructura y acción están inscritas en procesos de estructuración y no sometidos a dinámicas causales excluyentes. El agente solo puede ser entendido si se le ubica como parte de esa dualidad" (Ortiz 1999:77).

En contraposición, la propuesta analítica de Archer, basada en lo que denomina dualismo analítico, separa las dos dimensiones, estructura y agencia, analizándolas como dimensiones independientes (en términos teóricos), sin que ninguna se imponga o determine a la otra dimensión; pero también, reflexiona sobre el momento en que se da la interacción entre ambas. Para Archer, una de las ventajas de su enfoque propuesto es que permite dar cuenta de la influencia de cada dimensión, así como de las propiedades emergentes de cada una de ellas, mientras que el estructuracionismo giddensiano las rechaza, al tomar como elemento de análisis a la praxis, momento en el que se producen y reproducen las estructuras y se constituyen los actores.

Para Archer, la sociedad es concebida como el resultado emergente del juego mutuo entre estructura y agencia. Ni las presiones estructurales por sí mismas o la sola capacidad de agencia definirán el resultado de la acción, sino que ésta será resultado del arreglo específico que resulte in situ de la interpretación que el actor realice de las presiones estructurales, en conjunción con las relaciones de poder presentes en el contexto histórico de que se trate. Vale decir, que esta propuesta tiene como soporte la ontología estratificada de la realidad y su carácter de intransitividad, aporte del realismo crítico, en donde las propiedades emergentes de las estructuras y de los agentes son separables.

Por tanto, la concepción de realidad no se reduce a lo dado, debido a que, al encontrarse la realidad abierta, metodológicamente se tiene que dar cuenta de lo dado-dándose, a partir del análisis de cada una de las dimensiones, y también del juego mutuo que se establece entre ellas. Para ello, se tendrá que incorporar la variable teórica del tiempo, y así poder dar cuenta de la dinámica social. Así, Archer plantea al enfoque morfogenético, a partir del cual explica dicha dinámica.

El término de morfogénesis proviene de la teoría moderna de sistemas del sociólogo americano Walter Buckey, y se refiere a los procesos que tienden a elaborar o modificar una forma, una organización o un estado del sistema. Su contraparte, la morfostasis, implica los procesos de intercambio que tienden a preservar y mantener una forma, una organización o un estado del sistema. Archer utiliza estos dos 
procesos para analizar la interacción entre estructura y agencia, y explicar tanto la estabilidad como el cambio.

Para Archer: "el elemento 'morfo' es un reconocimiento al hecho de que la sociedad no tiene una forma predecidida o un estado preferido; la parte 'genética' es un reconocimiento a que la sociedad toma su forma y está formada por sus agentes, y que se origina a partir de las consecuencias no esperadas y no deseadas de sus actividades" (2009:32-33). Ello se vincula con la idea de lo social como un sistema abierto, cuyo carácter de innovación se lo da el hecho de estar poblado, en tanto que el poder de las personas está en su intencionalidad. Lo anterior forma parte de los presupuestos del realismo crítico.

Para explicar las modificaciones en la forma, o en las estructuras, el enfoque morfogenético considera tres fases: condicionamiento, interacción y elaboración. En este enfoque se incluye la variable teórica del tiempo, como se puede identificar a partir de las siguientes proposiciones (Archer 2009):

a) La estructura necesariamente antecede a las acciones que transforma y,

b) La elaboración estructural necesariamente viene después de tales acciones.

Dicho modelo parte de la separabilidad entre las dimensiones de análisis que se presentan, lo que posibilita "identificar discontinuidades temporales en el accionar de estructuras y agencias" (Parra 2016:224). Por lo tanto, el desfase temporal constituye la clave para una explicación de las propiedades de cada una de las dimensiones y del cambio social. Así, los procesos sociales están constituidos por secuencias morfogenéticas, dando como producto final la elaboración, misma que se constituye en condicionamiento de los sucesivos procesos.

De las relaciones duales que Archer va articulando con su propuesta teórico-metodológica, la distinción entre integración social e integración sistémica, propuesta por sociólogo británico David Lockwood, será central. Por integración social se entiende a las relaciones ordenadas o conflictivas entre actores, y por integración sistémica a las relaciones ordenadas o conflictivas entre partes de la estructura social. Para Lockwood, ambas formas de integración son analíticamente separables y remiten al problema de la agencia y la estructura, cuyo juego mutuo permite explicar la estabilidad y el cambio social.

Para Archer: "el dualismo analítico está en la base de la distinción fundamental de Lockwood entre integración sistémica y social, pero lo que quedó sin respuesta eran las preguntas cruciales sobre qué constituía los 'elementos componentes' de los sistemas sociales y cómo se podía sostener que ejercen efectos causales sobre las personas. El realismo social entregó la respuesta en términos de propiedades emergentes y sus poderes generativos" (2009:266). La explicación de estos componentes será desarrollada por Archer y abordada en este documento en el cuarto apartado.

\section{Enfoques de la cultura desde la sociología}

En este apartado, se revisan los enfoques de la cultura desde la sociología, a los cuales Archer critica con base en su modelo de dualismo analítico. La teoría de la cultura propuesta por Margaret Archer tiene como eje de discusión la relación cultura y agencia, la cual, a decir de ella misma, corre paralela al amplio debate acerca de la relación entre estructura y agencia, que ha tenido lugar dentro de la teoría social.

En el campo de la sociología, Archer distingue tres enfoques teóricos de la cultura, en los cuales se presenta la conflación, es decir, la incapacidad de dar cuenta del carácter estratificado de la realidad, al considerar 
solo la dimensión estructural o agencial. En el primer enfoque, de conflación descendente, la cultura es un macrofenómeno que guía a los actores. En el segundo, de conflación ascendente, un grupo de actores impone su visión del mundo a otros grupos minoritarios en términos de poder. El tercer enfoque, denominado conflación central o elisionismo, se centra en la integración entre el sistema cultural y la interacción cultural, pero no permite explicar la participación de cada una de las dimensiones, mismas que poseen cierta autonomía y diferentes temporalidades. Para un mejor entendimiento de estos tres enfoques, se presenta su caracterización.

\section{Conflacionismo descendente}

El enfoque de la cultura como un macrofenómeno que influye sobre los actores, se ejemplifica a través de la perspectiva estructuralista de Talcott Parsons. Dentro de esta perspectiva, la cultura se define como un sistema integrado por esquemas cognitivos de interpretación, formas simbólicas de expresión y estándares valorativos, que operan tanto a nivel micro como macro. A nivel micro, sirven de orientación al individuo en las interacciones socioculturales que se dan en la cotidianeidad, conduciendo a la complementariedad de expectativas entre alter y ego. A nivel macro, la función del sistema cultural consiste en mantener el orden y equilibrio dentro del sistema general, lo que se posibilita por la consistencia entre los diversos elementos del mundo social. Grosso modo, el sistema cultural es un sistema estructurado, integrado y homogéneo, que preexiste y subsiste al sujeto.

En esta concepción prevalece el mito de la integración cultural, tomado de la antropología en su fase inicial, denominada por Archer como conflacionismo descendente: "En la tradición conflacionista descendente (es decir, aquella que atribuye todo efecto causal al sistema) se supone que la coherencia del sistema cultural produce uniformidades a nivel de la mentalidad y la conducta de los miembros de una comunidad, produciendo por tanto cohesión social. Esta tradición ha desarrollado dos versiones del mito. En la versión del funcionalismo parsoniano la coherencia de las pautas culturales desciende a nivel de las instituciones y los roles y por medio de la internalización llega a las personalidades; el resultado es la cohesión. En la versión estructuralista, la idea central es que las culturas son códigos que producen las clasificaciones sociales que ordenan la vida social" (Murguía 2009:119-120).

Para la socióloga británica, el elemento central de la teoría de la cultura y del sistema de acción parsoniano lo constituye el consenso normativo, el cual se vincula directamente con la cohesión y el consenso. Sin embargo, la idea de consenso exime que el acuerdo normativo puede promover también el conflicto. Además, para Archer, una cosa es que el sistema cultural sea un sistema integrado, y otra que la gente se comporte de acuerdo con él. En la perspectiva de Archer no todo está integrado, pero sí vinculado, y ese vínculo puede ser de conflicto. El problema está en que el enfoque conflacionista descendente invisibiliza lo que ocurre a nivel de la interacción sociocultural; es decir, la capacidad agencial de resignificar las estructuras. Otra limitante de este enfoque es su incapacidad de explicar la dinámica cultural. Por lo tanto, bajo este planteamiento, Archer reconoce que la gente puede comportarse discordante con las normas socialmente aceptadas.

\section{Conflacionismo ascendente}

Si en el enfoque anterior el sistema cultural se interpone sobre la interacción sociocultural, en el conflacionismo ascendente ocurre lo opuesto. En este caso, este conflacionismo también es una fusión, donde la cultura se subsume en la agencia, puesto que considera dominante a la dimensión agencial. Aquí, los sujetos, o grupos de interés, tienen el protagonismo, toda vez que la cultura supone significados que 
solo pueden ser construidos por sujetos. En esta perspectiva se abandera la idea de que las clases dirigentes imponen un constructo cultural a todos los grupos sociales, como ocurrió con el clero, en la sociedad medieval, o con la burguesía, en la sociedad capitalista.

Es necesario precisar que, dentro de este enfoque, se pueden distinguir dos vertientes. La primera, que asume la idea de cultura dominante como algo homogéneo, que logra imponerse mediante diversos aparatos y mecanismos, logrando integrar a todos los grupos sociales a las ideas dominantes, y cancelando la posibilidad de cambio. La segunda vertiente reconoce la existencia de grupos subalternos, con culturas propias en disputa con la cultura dominante, lo que deja abierta la posibilidad de cambio. En ese tenor, a través del conflicto a nivel agencial se podrá transformar el sistema cultural dominante, con implicaciones en el sistema social.

Al respecto, la crítica de Archer al conflacionismo ascendente, en sus dos vertientes, se dirige al carácter unívoco que tienen acerca de la cultura y las clases dirigentes. En este enfoque conflacionista, se presenta a la cultura dominante como un todo integrado, sin contradicciones, que constriñe y se impone de manera homogénea sobre otros actores o grupos de interés.

A decir de Archer el conflacionismo ascendente tiene su raíz en una lectura reduccionista de Marx, y han sido algunos neomarxistas quienes han sostenido dicha postura. Empero, Archer, haciendo justicia a Marx, señala que él destacó problemas asociados con la conflación ascendente, los cuales se plantean a partir de las siguientes interrogantes: ¿Hay una sola cultura dominante? ¿La cultura dominante es internamente coherente? ¿Existen culturas subordinadas? ¿Cómo se produce la dominación cultural? Al respecto, Marx rechazó la unicidad de la cultura dominante, así como su consistencia lógica. También reconoció que existen otras culturas, como por ejemplo la campesina. Por último, consideró que la mistificación o universalización de las ideas de la clase dominante no ocurre de manera automática. En ese sentido, la propuesta de Marx dejó abierta una posibilidad de abordaje de la cultura bajo presupuestos distintos a los del mito de integración y univocidad de la cultura.

\section{Conflacionismo central}

Finalmente tenemos la postura de la conflación central, que Archer relaciona con el concepto de cultura de Giddens y de Bauman. Esta tercera perspectiva de la conflación hace referencia a la negativa de analizar por separado el sistema cultural y el nivel sociocultural, es decir, de abordarlas como dualidad. La siguiente cita ilustra su caracterización: "el núcleo de la concepción fusionista central de la cultura se refiere a su dualidad esencial. La cultura es el producto de la agencia humana, pero al mismo tiempo cualquier forma de interacción social está incorporada a ella: para Bauman, 'estar estructurado y ser capaz de estructurar parecen ser los núcleos gemelos del modo humano de vida conocido como cultura', para Giddens, 'la estructura es tanto el medio como el resultado de la reproducción de las prácticas'" (Archer 1988:111).

En esta postura, los fusionistas centrales asumen que todos los actores son participantes activos y al mismo tiempo reconocen la existencia de un sistema cultural de significados que sirve de medio para la acción, pero dan cuenta de ambos como dos caras de la misma moneda. Bauman, por ejemplo, en la introducción de su libro La cultura como praxis, al referirse al "mundo de la cultura", señala que "creatividad" y "regulación normativa" están imbricadas: "Ambas ideas no se pueden separar, sino que están presentes en la idea compuesta de cultura, y así deben permanecer" (Bauman 1999:22). Para el sociólogo polaco, la cultura pertenece a la familia de conceptos que representan la praxis humana. 
Acorde con estas ideas, Archer señala el interés que desde el conflacionismo central se tiene de abandonar los enfoques conflacionistas ascendente y descendente, al reconocer el carácter intersubjetivo de la cultura y, al mismo tiempo, la objetividad que ésta posee. Sin embargo, el inconveniente que Archer observa es que, no indagan sobre la actuación de cada dimensión (agencial y estructural), y la influencia que una ejerce sobre la otra. Así, esta perspectiva termina fusionando ambas a nivel central, al considerarlas como mutuamente constitutivas.

De esta manera, la crítica de Archer va en el sentido de que solo se observa al juego del sistema cultural y el sistema sociocultural en el momento de la praxis. Si uno se modifica se modifica el otro. Para Archer, el juego mutuo entre la cultura y la agencia es el fundamento de la dinámica cultural; sin embargo, considera que ambos tienen autonomía relativa y se mueven en una temporalidad distinta. Asimismo, cuestiona la incapacidad del conflacionismo central para responder a cuándo los actores pueden ser transformadores y cuándo son replicadores/reproductores.

\section{El enfoque morfogenético para el análisis cultural}

Para el análisis de la cultura, Archer se basa en el enfoque morfogenético, siguiendo la propuesta de los dualismos. Ella refiere la distinción entre el Sistema Cultural (SIC) y la Interacción Cultural (S-C), y su separabilidad analítica, de la siguiente forma: "Está claro que el Sistema Cultural y la vida Sociocultural no existen u operan independientemente uno del otro; se superponen, entrelazan e influyen recíprocamente. Pero ésta es precisamente la cuestión, dado que no afirmo el dualismo sino más bien la utilidad de un enfoque analíticamente dualista, cuya principal recomendación es el hecho mismo de que permite explorar este juego recíproco" (Archer 1988:16).

Bajo este supuesto, en el Sistema Cultural se dan relaciones lógicas entre proposiciones; mientras que, en la Interacción Cultural tienen lugar relaciones entre agentes. Archer puntualiza que la secuencia morfogenética se expresa a partir de lo siguiente (1988:140):

(i) Hay relaciones lógicas entre los componentes del Sistema Cultural (SIC).

(ii) Hay influencias causales ejercidas por el SIC sobre el nivel Sociocultural (S-C).

(iii) Hay relaciones causales entre grupos e individuos en el nivel S-C.

(iv) Hay elaboración del SIC debido a que el nivel S-C modifica las relaciones lógicas actuales e introduce otras nuevas.

Además, la propuesta de Archer incorpora, en el análisis de la cultura, la variable teórica del tiempo, y sostiene una crítica a los presupuestos que han sostenido el mito de la integración cultural. En su lugar, incorpora a la contradicción como principio ontológico, ya que considera que ésta se presenta en el SIC, en el S-C, y en la interacción entre ambos. Bajo este principio, las contradicciones y las complementariedades ideacionales del SIC son objetivas, es decir, existen al margen de la percepción que se tenga de ellas y son susceptibles de conocerse por referencia a principios lógicos.

La separabilidad analítica con la que Archer da cuenta de la configuración del SIC, y el reconocimiento que hace respecto a su existencia objetiva, se encuentra en oposición de quienes sostienen que los sistemas culturales no tienen una existencia independiente, que son socialmente relativos y que no se les puede examinar separadamente del S-C. 
Ahora bien, las contradicciones a nivel del SIC pueden ser coaccionantes en el S-C, cuando las ideas de un actor o grupo se encuentren en oposición o discrepancia con las ideas de otro actor o grupos, e intenten mantenerlas. Esta contradicción conllevará la necesidad de un arreglo específico a nivel del S-C.

Por su parte, el Sistema Sociocultural (S-C), viéndolo separadamente, no siempre está en armonía causal. Para Archer, un elemento fundamental de la Interacción Cultural son las relaciones de poder, debido a que el establecimiento de un sistema ideacional será resultado del arreglo que se establezca entre los actores intervinientes, en un tiempo y espacio determinado.

De esta forma, cuando en el S-C se logra percibir la contradicción lógica del SIC, en algunos casos se dará la manipulación, impidiendo que esta contradicción se visibilice, o se hará su corrección. La corrección se hará dependiendo de la correlación de fuerzas que se presente en el contexto de acción, imponiéndose la postura que tenga mayor fuerza, o en su defecto, se podrán corregir ambas para que sean recíprocamente consistentes. Como se observa, en el proceso de interacción, las relaciones causales no son independientes a las relaciones lógicas. Empero, la separabilidad analítica permite indagar en las propiedades y temporalidades que de manera específica presentan.

Debido a que uno de los objetivos de Archer es explicar teóricamente las condiciones para la estabilidad y el cambio cultural, es que recurre a la propuesta de Lockwood, en torno a la explicación de la integración social e integración sistémica. Esta propuesta la lleva al análisis de la cultura, y la traduce en la cohesión causal, hablando de la integración sociocultural; y en la consistencia lógica de la cultura, para explicar la integración del sistema cultural.

Así, la integración del sistema cultural implica compatibilidad entre los componentes ideacionales de la cultura, en tanto que se refiere al mundo de las ideas; mientras que la integración sociocultural supone uniformidad social, producida por la imposición de ideas, a partir de las relaciones de poder imperantes, por lo que se trata de efectos causales que son propiedad de las personas. Al respecto Archer prefigura las siguientes posibles combinatorias: integración sociocultural alta / integración del sistema cultural alta; integración sociocultural baja / integración del sistema cultural alta; integración sociocultural alta / integración del sistema cultural baja o integración sociocultural baja / integración del sistema cultural baja.

Siguiendo a Lockwood, Archer refiere que "ningún elemento por sí solo es condición suficiente para el cambio estructural" (1988:32). Y lo explica de la siguiente forma: "la integración Sistémica podrá ser baja, pero a menos que sus contradicciones fueran actualizadas y amplificadas por grupos sectoriales, se las podría contener y la estasis persistiría a causa de la elevada integración social. De manera alternativa, el antagonismo grupal podía ser profundo (baja integración social) sin conducir a cambios significativos en la sociedad, a menos que se asociara a contradicciones Sistémicas" (Archer 1988:32-33).

Esto implica que, pese al reconocimiento ontológico de la contradicción en cualquiera de las dimensiones del SIC o del S-C, existen períodos largos de estabilidad cultural y que, cuando ésta se rompe, se genera una nueva elaboración cultural. Con lo anterior, se puede afirmar que la propuesta de Archer establece un enfoque teórico-metodológico para el abordaje de la cultura alejado de la idea de cualquier determinismo.

\section{Comentarios finales}

Una de las grandes paradojas de la cultura, de acuerdo con las primeras perspectivas de abordaje, es su carácter estable, pero al mismo tiempo dinámico. Sin embargo, el enfoque morfogenético de la socióloga 
británica, Margaret Archer, es una propuesta de teorización sobre las condiciones para la estabilidad y el cambio cultural, a la vez que constituye una estrategia de abordaje que favorece la comprensión de aspectos que permanecen, y al mismo tiempo, de aspectos que van sufriendo modificaciones, vistos en una perspectiva longitudinal. La riqueza del planteamiento Archer está en la desmitificación de la integración cultural, y en la posibilidad de identificar elementos a partir de los cuales se puede explicar por qué algunas contradicciones producen cambios y otras no lo hacen.

Se trata de una propuesta que busca ser una alternativa a las posturas conflacionistas ascendente, descendente y central que Archer ubica en el análisis de la cultura, llevado a cabo desde la sociología. El eje de la propuesta de Archer se configura a partir del dualismo analítico y del enfoque morfogenético, ambos en articulación con los postulados del realismo crítico de Bashkar. Para ello, los fundamentos del realismo crítico constituyen un puente para entender la propuesta de Archer. En este caso, se refiere una noción de realidad abierta, por la intencionalidad de los sujetos que la pueblan; una realidad no predeterminada, es decir, contingente.

Como colofón, se debe señalar que, en una época carente de utopías en la que la sociedad se muestra unidimensional, la propuesta de Archer representa no solo una alternativa de análisis, sino que abre una posibilidad de otras direccionalidades en la realidad cultural. No solo aborda un problema teórico, también se confronta, a decir de ella misma, a un problema de carácter político acerca de la condición humana, reivindicando al hombre como sujeto.

\section{Bibliografía}

Archer, M. 1988. Cultura y teoría social. Buenos Aires: Nueva visión.

Archer, M. 2009. Teoría social realista: el enfoque morfogenético. Santiago: Ediciones Universidad Alberto Hurtado.

Bauman, Z. 1999. La cultura como praxis. Barcelona: Paidós Studio.

Marcareño, A. 2008. Acción, estructura y emergencia en la teoría sociológica. Revista de sociología 22: 217-256. https://doi.org/10.5354/0719-529X.2008.14492

Murguía, A. 2009. El análisis sociológico de la cultura. Teoría, significado y realidad después del giro lingüístico. México: UNAM.

Ortiz, L. 1999. Acción, significado y estructura en la teoría de A. Giddens. Convergencia 6(20): 57-84. http://www.redalyc.org/articulo.oa?id=10502002

Parada, J. 2004. Realismo crítico en investigación en ciencias sociales: una introducción. Investigación y Desarrollo 12(2): 396-429. http://www.redalyc.org/pdf/268/26810208.pdf

Parra, J. 2016. Realismo crítico: una alternativa en el análisis social. Revista Sociedad y Economía 31: 215238. http://www.redalyc.org/articulo.oa?id=99647007010

Recibido el 13 Ago 2017

Aceptado el 26 Oct 2017 